\title{
Lessons learned from SARS-CoV and MERS-CoV: FDA-approved Abelson tyrosine-protein kinase 2 inhibitors may help us combat SARS-CoV-2
}

\author{
Seyed Fazel Nabavi ${ }^{1}$, Solomon Habtemariam², Emilio Clementi ${ }^{3,4}$, Ioana Berindan-Neagoe ${ }^{5,6}$, \\ Cosmin Andrei Cismaru ${ }^{5,7}$, Mahsa Rasekhian ${ }^{8}$, Maciej Banach ${ }^{9,10}$, Morteza Izadi ${ }^{1}$, Mahdi Bagheri ${ }^{1}$, \\ Mohammad Sadegh Bagheri ${ }^{11}$, Seyed Mohammad Nabavi ${ }^{1}$
}

\author{
${ }^{1}$ Baqiyatallah Hospital, Baqiyatallah University of Medical Sciences, Tehran, Iran \\ ${ }^{2}$ Pharmacognosy Research Laboratories and Herbal Analysis Services, University \\ of Greenwich, Central Avenue, Chatham-Maritime, Kent ME4 4TB, United Kingdom \\ ${ }^{3}$ E. Medea Scientific Institute, Bosisio Parini, Italy \\ ${ }^{4}$ Unit of Clinical Pharmacology, Department of Biomedical and Clinical Sciences, \\ Luigi Sacco University Hospital, Università degli Studi di Milano, Milan, Italy \\ ${ }^{5}$ Research Centre for functional Genomics, Biomedicine, and Translational Medicine, \\ "Iuliu Hatieganu" University of Medicine and Pharmacy, Cluj-Napoca, Romania \\ ${ }^{6}$ The Research Centre for Advanced Medicine- Medfuture- "Iuliu Hatieganu" \\ University of Medicine and Pharmacy Cluj-Napoca, Romania \\ ${ }^{7}$ Department of Functional Sciences, Immunology, and Allergology, "luliu Hatieganu" \\ University of Medicine and Pharmacy Cluj-Napoca, Romania \\ ${ }^{8}$ Pharmaceutical Sciences Research Centre, Health Institute, Kermanshah University \\ of Medical Sciences, Kermanshah, Iran \\ ${ }^{9}$ Department of Hypertension, Chair of Nephrology and Hypertension, \\ Medical University of Lodz, Lodz, Poland \\ ${ }^{10}$ Polish Mother's Memorial Hospital Research Institute (PMMHRI), Lodz, Poland \\ ${ }^{11}$ Student Research Committee, Baqiyatallah University of Medical Sciences, Tehran, \\ Iran
}

Submitted: 9 April 2020

Accepted: 13 April 2020

Arch Med Sci 2020; 16 (3): 519-521

DOI: https://doi.org/10.5114/aoms.2020.94504

Copyright (c 2020 Termedia \& Banach

SARS-CoV-2 is a newly emerging infectious disease, which originated from Wuhan in the Hubei province of China in late December 2019 [1]. Since then, it has rapidly spread all over the world, and at the time of writing this letter, WHO statistics show more than 1,696,588 cases and 105,952 deaths confirmed across the world [2]. Although there is no specific therapy for SARS-CoV-2 infection [3], combination therapy with antiviral and anti-inflammatory drugs accompanied by supportive treatment have been used for SARS-CoV-2 patients [4]. The combination of well-known HIV protease inhibitors, such as ritonavir with lopinavir, has also been a common approach to treat SARS-CoV-2. Insufficient outcome in severe cases is, however, one of the main challenges associated with the current antiviral-based therapy for SARS-CoV-2 [5]. In view of the long period required for novel drug discovery and the desperate need for a prompt response to this pandemic infection, one must resort to repurposing FDA-approved drugs. In this direction, our experience with other close members of coronaviruses such as SARS and MERS has taught us that repurposing the current drugs is a reasonable strategy.

Abelson tyrosine-protein kinase 2 (Abl2), the imatinib target, was required for efficient SARS-CoV and MERS-CoV replication in vitro [6]. Coleman et al. have shown that the imatinib target $A b l 2$ is indispensable for efficient replication of SARS-CoV and MERS-CoV in vitro.
Corresponding authors:

Dr. Seyed Mohammad Nabavi

Baqiyatallah Hospital

Baqiyatallah University

of Medical Sciences

Tehran, Iran

E-mail: Nabavi208@gmail.com

Prof. Maciej Banach

Department

of Hypertension

Chair of Nephrology

and Hypertension

Medical University

of Lodz

Lodz, Poland

E-mail: maciejbanach77@

gmail.com 


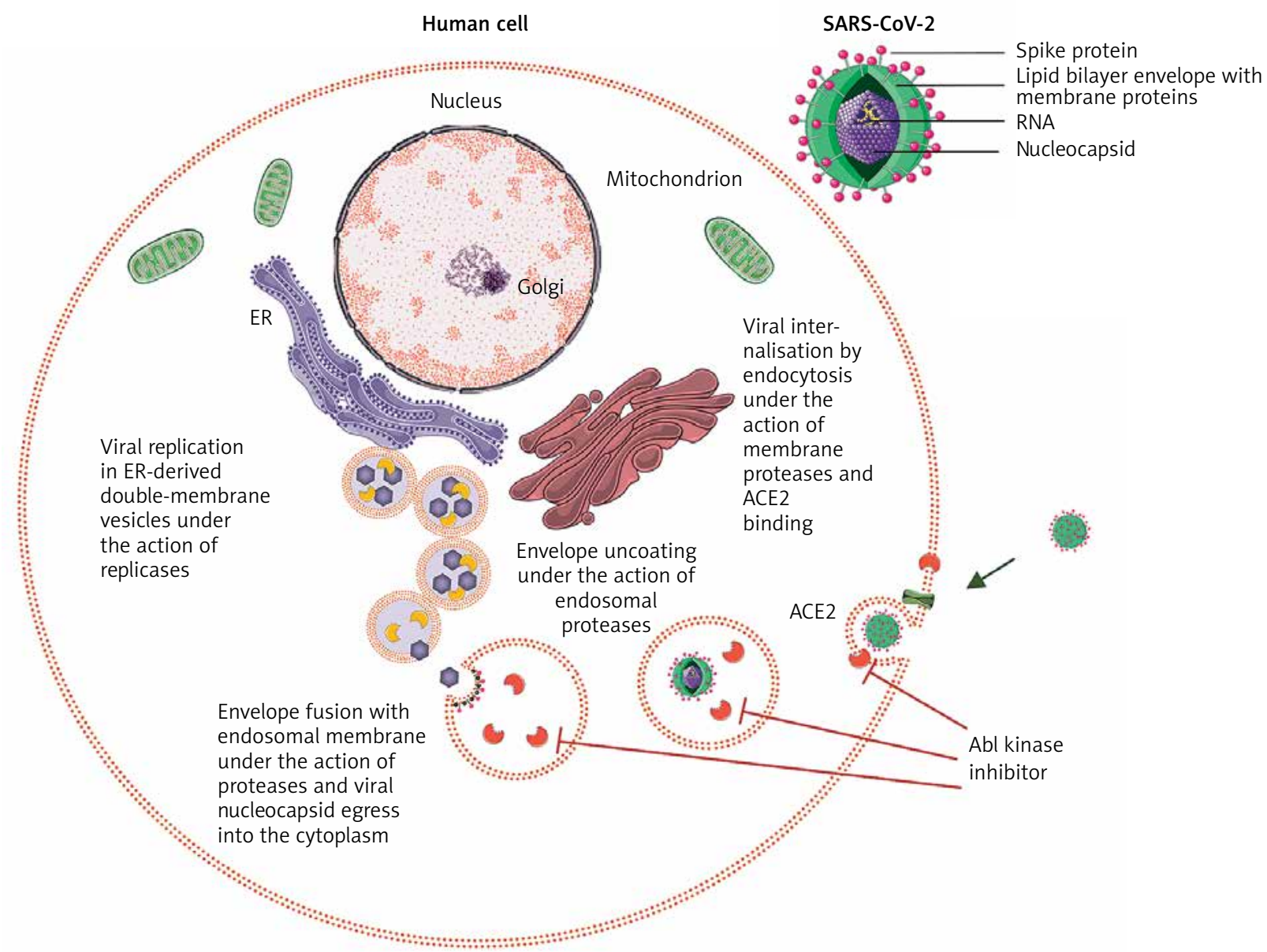

Figure 1. Abl kinase inhibition of early entry phases in the viral infectious cycle of SARS-CoV-2. Upon binding of SARS-CoV-2 spike protein to ACE2, the action of proteases at the cell membrane and in the endosomal compartment is required to complete the subsequent fusion steps of the virus. Abl kinase inhibitors act on proteases required for the completion of these steps by blocking their activity in SARS-CoV and MERS-CoV infection in vitro, and a similar activity of proteases is seen in SARS-CoV-2

Abl kinase activity and associated pathways have been shown to be involved in the coronavirus fusion step with endosomal membrane as well as the cell-cell fusion that occurs late in infection [7]. The authors have shown that imatinib and two specific Abl kinase inhibitors (GNF2 and GNF5) could reduce infectious bronchitis virus (IBV) titres by blocking the early entry phase including the S-induced syncytia formation prior to the hemifusion step.

Saracatinib, an inhibitor of multiple members of the Src family of tyrosine kinases (SFK) (Fyn/ Lyn), together with Abl2 kinase, interferes with the early stages of the MERS-CoV life cycle after internalisation into Huh-7 cells [8]. Because the SFKs regulate multiple signalling pathways, including EGF receptor (EGFR), Ras/Raf/MEK, PI3K/ AKT, and JAK/STAT, the role of Abl kinase inhibition by saracatinib in the observed antiviral effect is not clear. A plethora of evidences support the idea of repurposing kinase inhibitors for inhibition of viral replication.
Although there are differences in the transmission and mortality rates, there are many similarities in the genetics, pathogenesis, clinical manifestation, etc. between SARS-CoV, MERS- Cov, and SARSCoV-2 [9]. Previous valuable lessons learned from other coronavirus outbreaks and a plethora of research data on this topic can help us select the best treatment strategy for managing the 2020 pandemic. Taken together, repurposing the FDA-approved Abl2 inhibitors imatinib and saracatinib should be clinically tested for their antiviral effects in the early stage of SARS-CoV-2 infection, either alone or in combination with current antiviral drugs (except ritonavir, due to its interaction with imatinib).

\section{Conflict of interest}

The authors declare no conflict of interest.

\section{References}

1. Wang LS, Wang YR, Ye DW, Liu QQ. A review of the 2019 Novel Coronavirus (COVID-19) based on current 
evidence. Int J Antimicrob Agents 2020; 105948. doi: 10.1016/j.ijantimicag.2020.105948.

2. World Health Organization. Coronavirus disease 2019 (COVID-19): situation report, 83. 2020.

3. Chen L, Xiong J, Bao L, Shi Y. Convalescent plasma as a potential therapy for COVID-19. Lancet Infect Dis 2020; 20: 398-400.

4. Stebbing J, Phelan A, Griffin I, et al. COVID-19: combining antiviral and anti-inflammatory treatments. Lancet Infect Dis 2020; 20: 400-2.

5. Cao B, Wang Y, Wen D, et al. A trial of lopinavir-ritonavir in adults hospitalized with severe Covid-19. N Engl J Med 2020; doi: 10.1056/NEJMoa2001282.

6. Coleman CM, Sisk JM, Mingo RM, Nelson EA, White JM, Frieman MB. Abelson kinase inhibitors are potent inhibitors of severe acute respiratory syndrome coronavirus and middle east respiratory syndrome coronavirus fusion. J Virol 2016; 90: 8924-33.

7. Sisk JM, Frieman MB, Machamer CE. Coronavirus S protein-induced fusion is blocked prior to hemifusion by Abl kinase inhibitors. J Gen Virol 2018; 99: 619-30.

8. Shin JS, Jung E, Kim M, Baric RS, Go YY. Saracatinib inhibits middle east respiratory syndrome-coronavirus replication in vitro. Viruses 2018; 10: 283.

9. Katsiki N, Banach M, Mikhailidis DP. Lipid-lowering therapy and renin-angiotensin-aldosterone system inhibitors in the era of the COVID-19 pandemic. Arch Med Sci 2020; 16: 485-9. 